\title{
Non- surgical healing of a Large Cyst-Like Periradicular Lesion Using local drug delivery: A Case Report
}

\author{
${ }^{1}$ Dr. Darshan Shah, ${ }^{2}$ Dr. N. U. Manwar, ${ }^{3}$ Dr. Manoj Chandak, ${ }^{4}$ Dr. Abhay John, \\ ${ }^{5}$ Dr. Rushabh Malde \\ ${ }^{I}$ Post Graduate Student, Dept. of Conservative Dentistry and Endodontics, SharadPawar Dental \\ College,Sawangi(Meghe), Wardha \\ ${ }^{2}$ Professor, Dept. of Conservative Dentistry and Endodontics, SharadPawar Dental College,Sawangi(Meghe), \\ Wardha. \\ ${ }^{3}$ Prof and Head, Dept. of Conservative Dentistry and Endodontics, SharadPawar Dental \\ College,Sawangi(Meghe), Wardha. \\ ${ }^{4}$ Assistant Professor, Dept. of Pharmacology, Rural Medical College, LoniBk \\ ${ }^{5}$ Private Dentist, Mumbai
}

\begin{abstract}
This casereport describes the endodontic management of a large cyst-like periradicular lesion using a combination of antibiotic drugs. A 18-yr-old girl with a large cyst-like lesion from the apices of her teeth no 11 and 21. During treatment procedure, $2.5 \%$ sodium hypochlorite solution and $2 \%$ chlorhexidine gluconate was used for irrigation and a combination of antibiotic drugs was used for the intra canal dressing. Obturation was done after2-month and periapical healing was observed after 6 months
\end{abstract}

Keywords: Periapical lesion, Endodontic therapy, Lesion sterilization and tissue repair

\section{Introduction}

Conventional root canal therapy aims at removal of bacteria by filing or using chemical irrigation. When large periradicular lesions are present, however successful outcome is based on the removal of the residual bacteria present in the deeper layers of the dentine which may not be removed by conventional therapy,thereby necessitating additional treatment options to bring about resolution of such lesions. Various treatment modalities ranging from non-surgical retreatment, to rule out treatment inadequacies or presence of morphological abnormalities, to surgical treatment including curettage and apical resection have been considered. For large cystic lesions, however, simpler approaches such as marsupialization or tube decompression have been suggested to be viable alternatives (Hoen et al 1990).Most periapical lesions $(>90 \%)$ can be classified as either dental granulomas,radicular cysts or abscess (Bhaskar 1966,Lalonde\&Luebke 1968). Although differentiation of cysts from periapical granulomas is difficult radiographically( Natkin et al1984), studies relating radiographic lesion size to histology have stated that with a lesion size of $200 \mathrm{~mm}$ or larger, the incidence of cysts was almost $100 \%$.It has been stated that lesions having an intact epithelial lining may not heal by non-surgical therapy since such lesions may have developed into a self-perpetuating entity (apical true cyst, Nair 1998). On other occasions, a large periapical lesion may have a direct communication with the root canal system (apical pocket cyst, Nair 1998) and respond favourably to non-surgical treatment (Hoen et al. 1990). In recent years, the concept of 'Lesion sterilization and tissue repair (LSTR) therapy' ${ }^{1,2}$ that employs the use of a combination of antibacterial drugs for disinfection of oral infectious lesions, including dentinal, pulpal, and periradicular lesions has evolved. According to this concept a repair of the damaged tissues may be brought about by disinfection of the lesions. ${ }^{3}$ The following case report describes the endodontic treatment of traumatized maxillary incisors which were associated with a large periapical lesion using LSTR therapy.

\section{Case Report}

A 18-year-old female patient was referred to the Department of Conservative Dentistry and Endodontics with a chief complaint of discoloration of maxillary left central incisor teeth(Fig 1). A history of trauma was reported when the patient was aged 10 years after the incident she did not visit a dentist as she didn't have any pain.. During her growing years upto 15 years, she occasionally experienced mild pain , but didn't have any evidence of swelling in her maxillary anterior region. On clinical examination there were no signs of scarring or fistulae of the soft tissues. Her left central incisor was discolored. Both the central incisors were slighty tender but there was no mobility. Maxillary right and left central incisor teeth did not respond to electric pulp testing.Radiographs demonstrated a large radiolucent lesion with a well-defined margin around the apex of the maxillary left central incisor(Fig 2). The maxillary left central incisor was anaesthetized and isolated with rubber dam.Following access cavity preparation, necrotic pulp tissue was extirpated. Pus was drained through 
the root canals. When drainage was ceased,the working length was determined (Fig 3) and the canals were prepared about $1 \mathrm{~mm}$ short of the radiographic apices with size 15-80 K-files and 15-60 K- files using a stepback technique. Copious irrigation was done with $2 \%$ chlorhexidine gluconate and saline. Intracanal medicament was not applied in the canals until drainage ceased. Final instrumentation was done and canals were copiously irrigated with $2.5 \%$ sodium hypochlorite solution. After drying the canals with sterile paper points, a mixture of Metronidazole, Ciprofloxacin and Minocycline paste as described by Takushige et al. was prepared into a creamy consistency and applied down the canals with the help of a lentulospiral .( Fig 3,4,5,6,7)The patient was recalled after 2 months and on examination the area revealed significant healing. The tooth was asymptomatic.The antibiotic paste was removed at this appointment. Final irrigation with $2 \%$ chlorhexidine gluconate and the root-canals were obturated with gutta-percha and AH 26 sealer (Dentsply/DeTrey, Konstanz, Germany) using a lateral condensation technique (Fig 8).After 3 weeks, non-vital bleaching was done with tooth no 21.The access cavity was sealed with composite(Ceram X Duo, Dentsply, Konstanz, Switzerland) and the patient was recalled after 6 months. The radiographic examination revealed significant healing, the absence of apical radiolucency and presence of bony trabeculae(Fig 9). Clinically there was no sensitivity and pain to percussion or palpation and the soft tissues were in a healthy state.

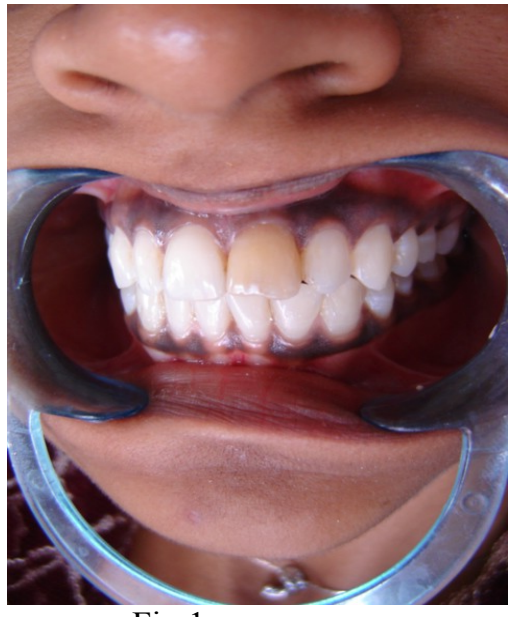

Fig 1

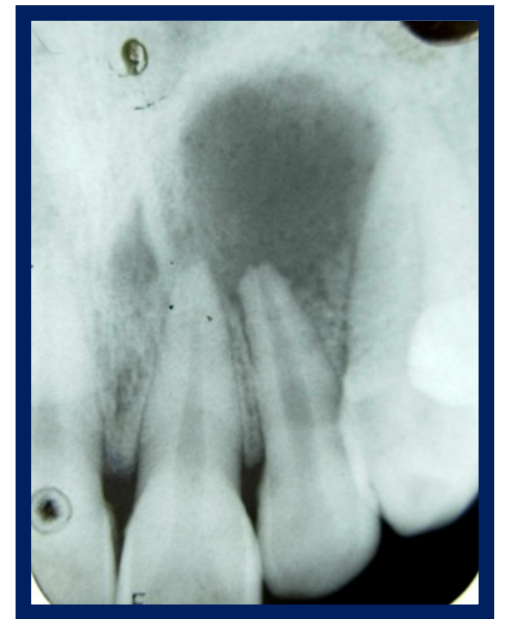

Fig 2

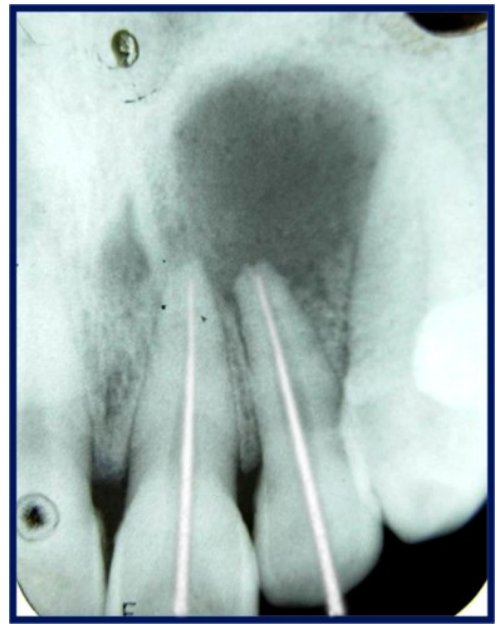

Fig 3

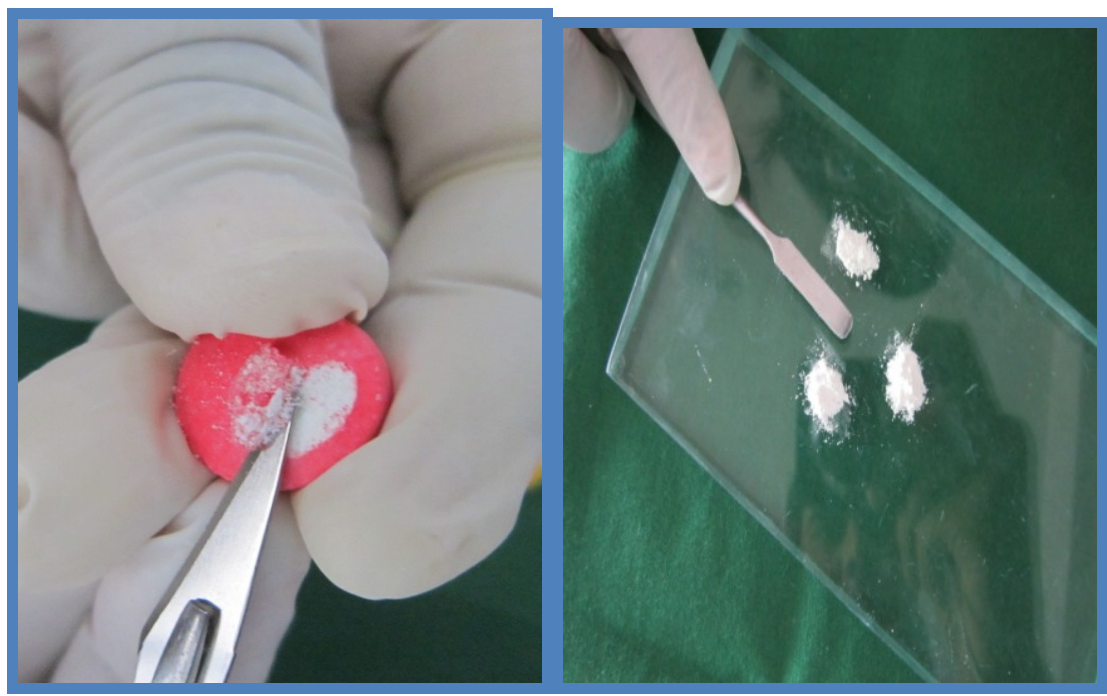

Fig 4

Fig 5 


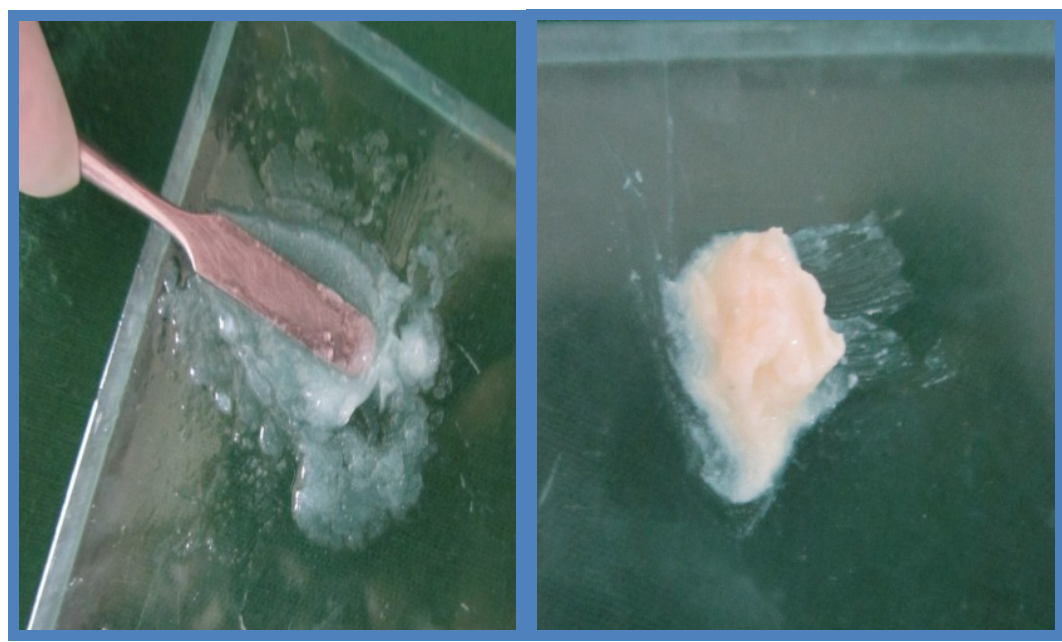

Fig 6

Fig 7

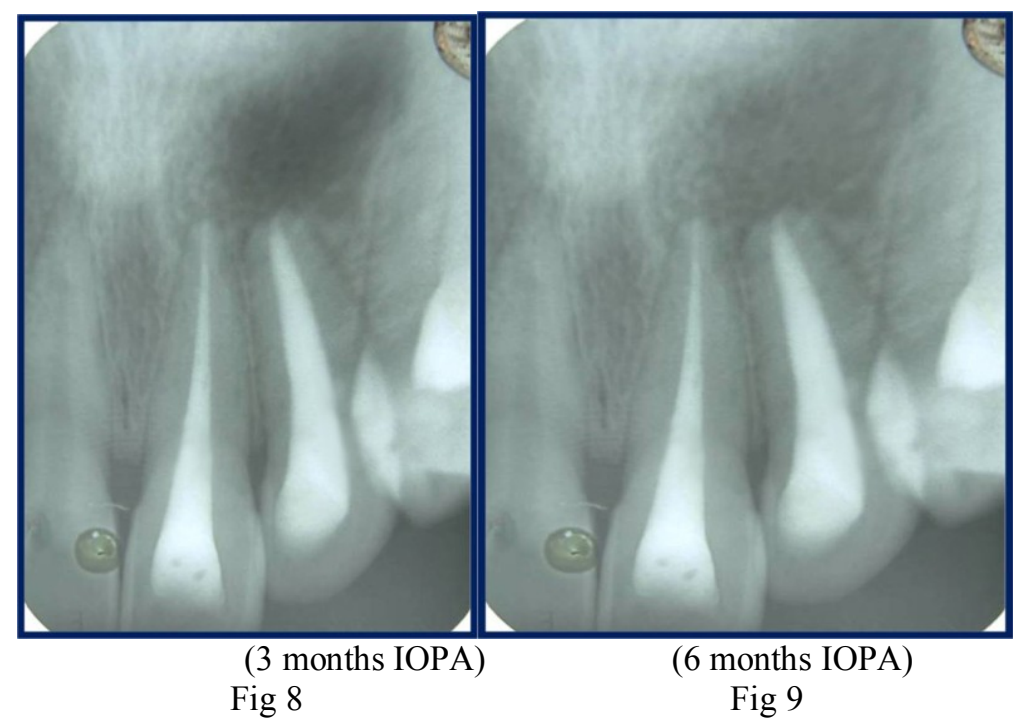

III. Discussion

The response to trauma can be varied. Some pulps remain apparently normal with no adverse effects, whereas others become necrotic. Necrotic pulps provide a good nutritional supply for pathogenic bacteria, which must be present for the development of a periapical lesion. In some cases periapical inflammation begins before the pulp is totally necrotic, and it is possible to have periapical radiolucency despite the presence of some vital tissue remaining in the root canal (Sundqvist 1976, Khayat et al. 1988). The definitive diagnosis of the type of periradicular lesion can only be made by a histological examination. ${ }^{17}$ However, a preliminary clinical diagnosis of a periradicular cyst is reasonable if all of the following conditions exist: (a) the periradicular lesion involves one ormore teeth with necrotic pulps; (b) the lesion is greater than $200 \mathrm{~mm} 2$ in size; (c) a straw-colored fluid is produced upon aspiration or on drainage through an access; and (d) the fluid contains cholesterol crystals ${ }^{18}$. Additionally, the incidence of cysts has been shown to be 60 to $67 \%$ in lesions with a diameter of 10 to 20 $\mathrm{mm}{ }^{19}, 20$. Cholesterol crystals are encountered in 29 to $43 \%$ of dental cysts ${ }^{21}$. They are more frequently encountered in radicular cysts than in apical granulomata. Crystals are identifiable under a microscope ${ }^{22}$. The treatment options available to manage large cysts range from nonsurgical root canal treatment and/or apical surgery to extraction. In some instances, nonsurgical treatment may be ineffective or difficult; those cases may be treated by surgery. In the present study, radiographs revealed that the involved teeth had large periradicular lesion with uniformly dense radiolucency and well-defined margins around the apices. Matsumoto et al. ${ }^{23}$ has demonstrated that the prognosis for the treatment of large periradicular lesions is not as good as that of small lesions. In contrast Strindberg ${ }^{24}$ and Sjogren et al. ${ }^{25}$ found no significant differences in healing frequency between lesions initially larger than $5 \mathrm{~mm}$ and those smaller than $5 \mathrm{~mm}$. They have also stressed the importance of a long observation time for treated teeth with Periradicular lesions. In a long time period clinical study, Calskan ${ }^{17}$ had reported 42 non-surgically treated teeth with large cyst-like lesions out of which $73.8 \%$ of all cases completely healed with nonsurgical treatment. It was demonstrated in this case report that the use of a 
combination of antibiotic drugs in tooth with large cyst-like periradicular lesion gave excellent clinical results. Repair of damaged tissues can be expected if lesions are disinfected ${ }^{3}$. In studies ${ }^{4-6}$, oral lesions have been analyzed under strict anaerobic conditions to understand the target bacteriain endodontic treatment. In addition, bacteria in dental plaque ${ }^{7}$, tongue plaque ${ }^{8}$, denture plaque ${ }^{9}$, saliva ${ }^{10}$, periodontal pockets ${ }^{11}$, and osteomyelitis ${ }^{12}$ have also been analyzed with the strict anaerobic procedures because they were possible sources of bacteria in endodontic lesions. According to these studies antibacterial drugs were selected. Metronidazole was the first choice because it has a wide bactericidal spectrum against anaerobes ${ }^{13}$, which were common in oral sites. However, some bacteria in lesions were resistant to metronidazole and, thus, two other antibacterial drugs, e.g., ciprofloxacin and minocycline, should be mixed with metronidazole ${ }^{14}$ in an effort to eliminate all the bacteria. Finally, extensive in vitro and in situ studies have been conducted showing the mixed drugs to be effective against oral bacteria ${ }^{3,15,16}$. Previous studies ${ }^{3,14-16}$ have clearly demonstrated that this combination is capable of eliminating bacteria from infected dental tissues.Caution should be taken in general when dentists give local or systemic drugs. Although the volumes of the drugs applied in this therapy are small and there were no reports of side effects, care should be taken if patients are sensitive to chemicals or antibiotics.

\section{Conclusion}

Root canal treatment using a combination of antibiotic drugs as an antibacterial dressing was successful in healing large cyst-like Periradicular lesion.

\section{References}

[1]. Iwaku M, Hoshino E, Kota K. Lesion sterilization and tissue repair (LSTR) therapy: new pulpal treatment. How to conserve infected pulps. Tokyo, Japan: Nihon-Shika- Hyoron, 1996.

[2]. Hoshino E, Takushige T. LSTR 3Mix-MP method-better and efficient clinical procedures of lesion sterilization and tissue repair (LSTR) therapy. Dent Rev 1998;666: 57-106.

[3]. Takushige T, Cruz EV, Asgor Moral A, Hoshino E. Endodontic treatment of primary teeth using a combination of antibacterial drugs. IntEndod J 2004;37:132-8.

[4]. Hoshino E Ando N, Sato M, Kota K. Bacterial invasion of non-exposed dental pulp. Int Endod J 1992;25:2-5.

[5]. Ando N, Hoshino E. Predominant obligate anaerobes invading the deep layers of root canal dentine. IntEndod J 1990;23:20 -7.

[6]. Kiryu T, Hoshino E, Iwaku M. Bacteria invading periapicalcementum. J Endod 1994; 20:169 -72.

[7]. Hoshino E, Sato M, Sasano T, Kota K. Characterization of bacterial deposits formed invivo on hydrogen-ion-sensitive field transistor electrodes and enamel surfaces. Jpn J Oral Biol 1989;31:102-6.

[8]. Hori R, Kohno S, Hoshino E. Tongue microflora in edentulous geriatric denturewearers. Microb Ecol Health Dis 1999;11:89 -95.

[9]. Hoshino E, Sato M. Composition of bacterial deposits on full denture. Jpn J Prosth Dent 1988;32:762-6.

[10]. Sato M, Hoshino E, Nomura S, Ishioka K. Salivary microflora of geriatric edentulous persons wearing dentures. Microb Ecol Health Dis 1993;6:293-9.

[11]. Uematsu H, Hoshino E. Predominant obligate anaerobes in human periodontal pockets. J Periodontal Res 1992;27:15-9.

[12]. Hoshino E, Echigo S, Yamada T, Teshima T. Isolation of Propionibacterium acnes fromsclerosing osteomyelitis of mandibles. Jpn J Oral Biol 1984;26:48 -51.

[13]. Ingham HR, Selkon JB, Hale JH. The antibacterial activity of metronidazole. J Antimicrob Chemother 1975;1:355-61.

[14]. Sato T, Hoshino E, Uematsu H, Noda T. In vitro antimicrobial susceptibility to combinations of drugs of bacteria from carious and endodontic lesions of human deciduous teeth. Oral MicrobiolImmunol 1993;8:172-6.

[15]. Hoshino E, Kurihara-Ando N, Sato I, et al. In vitro antibacterial susceptibility of bacteria taken from infected root dentine to a mixture of ciprofloxacin, metronidazole and minocycline. IntEndod J 1996;29:125-30.

[16]. Sato I, Kurihara-Ando N, Kota K, Iwaku M, Hoshino E. Sterilization of infected rootcanal dentine by topical application of a mixture of ciprofloxacin, metronidazole and minocycline in situ. IntEndod J 1996;29:118 -24.

[17]. C, als, kan MK. Prognosis of large cyst-like periapical lesions following nonsurgical root canal treatment: a clinical review. IntEndod J 2004;37:408 - 16.1

[18]. Eversole RL. Clinical outline of oral pathology: diagnosis and treatment, 2nd ed. Philadelphia, PA: Lea \&Febiger, 1984;203-59.

[19]. Lalonde ER. A new rationale for the management of the periapical granulomas and cysts: an evaluation of histopathological and radiographic findings. J AmDental Assoc 1970;80:1056 -9.

[20]. Morse DR, Patnik JW, Schacterle GR. Electrophoretic differentiation of radicular cysts and granulomas. Oral Surg Oral Med Oral Pathol 1973;35:249-64.

[21]. Browne RM. The origin of cholesterol in odontogenic cysts in man. Arch Oral Biol 1971;16:107-13.

[22]. Matsumoto T, Nagai T, Ida K, et al. Factors affecting successful prognosis of root canal treatment. J Endod 1987;13:239-42.

[23]. Strindberg LZ. The dependence of the result of pulp therapy on certain factors. An analytic study based on radiographic and clinical follow-up examinations. ActaOdontol Scand 14(Suppl 21):1-175, 1956.

[24]. Sjogren U, Hagglund B, Sundqvist G, Wing K. Factors affecting the long-term results of endodontic treatment. J Endod $1990 ; 16: 498-504$. 


\begin{abstract}
Authors
Dr. Darshan Shah Post Graduate Student, Dept. Of Conservative Dentistry and Endodontics, Sharad Pawar Dental College, Sawangi( Meghe), Wardha.
\end{abstract}

Dr. N. U. Manwar, Professor, Dept. Of Conservative Dentistry and Endodontics, Sharad Pawar Dental College, Sawangi( Meghe), Wardha.

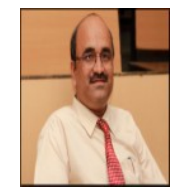

Dr. Manoj Chandak „Prof And HOD, Dept. Of Conservative Dentistry and Endodontics, Sharad Pawar Dental College, Sawangi( Meghe), Wardha

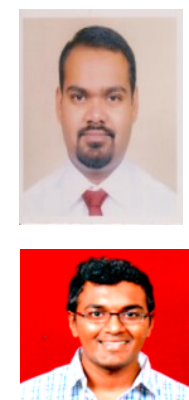

Dr. Abhay John, Assistant Professor, Dept. of Pharmacology, Rural Medical College, Loni Bk

Dr. Rushabh Malde, Private Dentist,

Mumbai 